\title{
Graph Cuts Framework for Kidney Segmentation with Prior Shape Constraints
}

\author{
Asem M. Ali ${ }^{1}$, Aly A. Farag ${ }^{1}$, and Ayman S. El-Baz ${ }^{2}$ \\ ${ }^{1}$ CVIP Laboratory, University of Louisville, USA \\ asem, farag@cvip.uofl.edu \\ ${ }^{2}$ Bioengineering Dept., University of Louisville, USA \\ aselba01@louisville.edu
}

\begin{abstract}
We propose a novel kidney segmentation approach based on the graph cuts technique. The proposed approach depends on both image appearance and shape information. Shape information is gathered from a set of training shapes. Then we estimate the shape variations using a new distance probabilistic model which approximates the marginal densities of the kidney and its background in the variability region using a Poisson distribution refined by positive and negative Gaussian components. To segment a kidney slice, we align it with the training slices so we can use the distance probabilistic model. Then its gray level is approximated with a LCG with sign-alternate components. The spatial interaction between the neighboring pixels is identified using a new analytical approach. Finally, we formulate a new energy function using both image appearance models and shape constraints. This function is globally minimized using $s / t$ graph cuts to get the optimal segmentation. Experimental results show that the proposed technique gives promising results compared to others without shape constraints.
\end{abstract}

\section{Introduction}

Isolating the kidney from its surrounding anatomical structures is a crucial step in many unsupervised frameworks that assess the renal functions, such as frameworks that are proposed for automatic classification of normal kidneys and acute rejection transplants from Dynamic Contrast Enhanced Magnetic Resonance Imaging (DCE-MRI). Many techniques were developed for kidney segmentation, Priester et al. 1] subtracted the average of precontrast images from the average of early-enhancement images, and black-and-white kidney mask is generated by a threshold. This mask image is eroded and the kidney contour is obtained with help of manual interactions. Giele et al. 2] improved the previous technique by applying an erosion filter to the mask image to obtain a contour via a second subtraction stage. A hull function is used to close possible gaps in this contour, then via repeated erosions applied to this contour, several rings were obtained, which formed the basics of the segmentation of the cortex from the medulla structures. Boykov et al. 3. used graph cuts to get a globally 
optimal object extraction method for dynamic N-data sets. They minimized cost function, which combined region and boundary properties of segments as well as topological constraints. Although the results looked promising, manual interaction was still required. Sun et al. introduced many computerized schemes for kidney segmentation and registration. In [4, after roughly alinement, they subtracted a high-contrast image from a pre-contrast image to obtain a kidney contour, which was propagated over the other frames searching for the rigid registration parameters. They used the level sets to segment the cortex and medulla. Most of these previous works, analyzed healthy transplants images. However, poor kidney function decreases the uptake of contrast agent, resulting in disjoined bright regions, so edge detection algorithms generally failed in giving connected contours.

The literature is rich with another segmentation approaches: simple techniques (e.g. region growing or thresholding), parametric deformable models and geometrical deformable models. However, all these methods tend to fail in the case of noise, gray level inhomogeneities, and diffused boundaries. In the area of medical imaging, organs have well-constrained forms within a family of shapes [5]. Therefore segmentation algorithms have to exploit the prior knowledge of shapes and other properties of the structures to be segmented. Leventon et al. 6 . combine the shape and deformable model by attracting the level set function to the likely shapes from a training set specified by principal component analysis (PCA). To make the shape guides the segmentation process, Chen et al. 7] defined an energy functional which basically minimizes an Euclidean distance between a given point and its shape prior. Huang et. al. 8], combine registration with segmentation in an energy minimization problem. The evolving curve is registered iteratively with a shape model using the level sets. They minimized a certain function to estimate the transformation parameters. In Paragios's work [9], a shape prior and its variance obtained from training data are used to define a Gaussian distribution, which is then used in the external energy component of a level sets framework.

In this paper, we propose a new kidney segmentation approach that uses graph cuts to combine region and boundary properties of segments as well as shape constraints. We generate from a set of kidney aligned images an image consisting of three segments: common kidney, common background, and shape variability region. We model the shape variations using a new distance probabilistic model. This distance model approximates the distance marginal densities of the kidney and its background inside the variability region using a Poisson distribution refined by positive and negative Gaussian components. For each given kidney slice, to use the distance probabilistic model, we align the given image with the training images. Then its gray level is approximated with a linear combination of Gaussian distributions (LCG) with positive and negative components. Finally, we globally minimized a new energy function using $s / t$ graph cuts to get the optimal segmentation. This function is formulated such that it combines region and boundary properties, and the shape information. 


\section{Proposed Segmentation Framework}

Recently, graph cuts appeared as a powerful optimization technique to get the optimal segmentation because it optimizes energy functions that can integrate regions and boundary properties of segments. The weighted undirected graph is a set of vertices, and a set of edges connecting these vertices. Each edge is assigned a nonnegative weight. The set of vertices corresponds to the set of image pixels $\mathcal{P}$, and two specially terminal vertices $s$ (source), and $t$ (sink). An example for the graph that we used in kidney segmentation is shown in Fig. 4. Consider a neighborhood system in $\mathcal{P}$, which is represented by a set $\mathcal{N}$ of all unordered pairs $\{p, q\}$ of neighboring pixels in $\mathcal{P}$. Let $\mathcal{L}$ the set of labels $\{$ " $0 "$, " $1 "\}$, correspond to kidney and background, respectively. Labelling is a mapping from $\mathcal{P}$ to $\mathcal{L}$, and we denote the set of labelling by $\mathbf{f}=\left\{f_{p}: p \in \mathcal{P}, f_{p} \in \mathcal{L}\right\}$. Now our goal is to find the optimal segmentation, best labelling $\mathbf{f}$, by minimizing a new energy function which combines region and boundary properties of segments as well as shape constraints. This function is defined as follows:

$$
E(\mathbf{f})=\sum_{p \in \mathcal{P}} S\left(f_{p}\right)+\sum_{p \in \mathcal{P}} D\left(f_{p}\right)+\sum_{\{p, q\} \in \mathcal{N}} V\left(f_{p}, f_{q}\right),
$$

where $S\left(f_{p}\right)$ measures how much assigning a label $f_{p}$ to pixel $p$ disagrees with the shape information, this will be explained in Sec. 2.1. $D\left(f_{p}\right)$ measures how much assigning a label $f_{p}$ to pixel $p$ disagrees with the pixel intensity $I_{p} . V\left(f_{p}, f_{q}\right)$ represents the penalty of the discontinuity between pixels $p$ and $q$. The last two terms will be explained in Sec. 2.2.

\subsection{Shape Model Construction}

Kidney shape model is created from a training set of kidney DCE-MRI slices. Fig 1 illustrates the steps used to create the shape model. Fig 1(a) shows a sample of the DCE-MRI kidney slices. First, we manually segment the kidneys (by a radiologist), as shown in Fig,1(b). Then the segmented kidneys are aligned using 2D rigid registration 10, see Fig[1(c). The aligned images are converted to binary images, as shown in Fig[1(d). Finally, we generate a "shape image" $\mathcal{P}_{s}=\mathcal{K} \cup \mathcal{B} \cup \mathcal{V}$ as shown in Fig2(a). The white color represents $\mathcal{K}$ (kidney), black represents $\mathcal{B}$ (background), and gray is the variability region $\mathcal{V}$. To model the shape variations, variability region $\mathcal{V}$, we use a distance probabilistic model. The distance probabilistic model describes the object (and background) in the variability region as a function of the normal distance $d_{p}=\min _{c \in \mathbf{C}_{\mathcal{K} \mathcal{V}}}\|p-c\|$ from a pixel $p \in \mathcal{V}$ to the kidney/variability contour $\mathbf{C}_{\mathcal{K} \mathcal{V}}$. Each set of pixels located at equal distance $d_{p}$ from $\mathbf{C}_{\mathcal{K} \mathcal{V}}$ constitutes an iso-contour $\mathbf{C}_{d_{p}}$ for $\mathbf{C}_{\mathcal{K} \mathcal{V}}$ as shown in Fig2(b) (To clarify the iso-contours, we enlarge the variability region without scale). To estimate the marginal density of the kidney, we assume that each isocontour $\mathbf{C}_{d_{p}}$ is a normally propagated wave from $\mathbf{C}_{\mathcal{K} \mathcal{V}}$. The probability of an iso-contour to be object decays exponentially as the discrete index $d_{p}$ increases. So we model the distance histogram by a Poisson distribution. We estimate the 


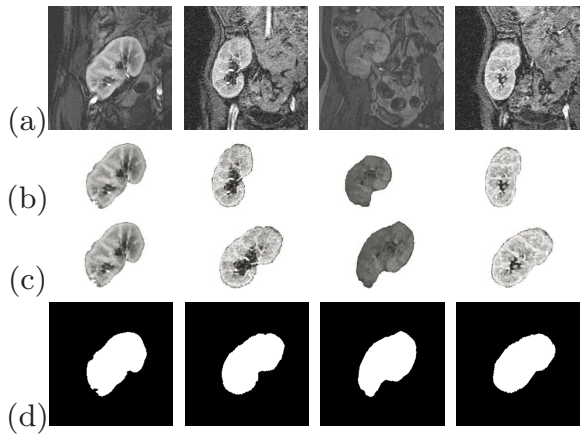

Fig. 1. Samples of kidney training data images: (a)Original , (b)Segmented , (c)Aligned (d)Binary

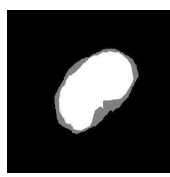

(a)

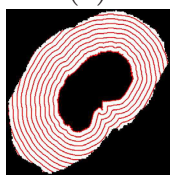

(b)

Fig. 2. (a) The labelled image, (b) The iso-contours
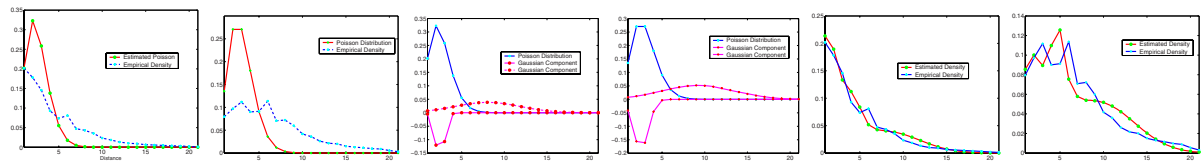

Fig. 3. [a,b]Empirical densities and the estimated Poisson distributions, [c,d] Components of distance probabilistic models, [e,f] Final estimated densities

kidney distance histogram as follows. The histogram entity at distance $d_{p}$ is defined as

$$
h_{d p}=\sum_{i=1}^{M} \sum_{p \in \mathbf{C}_{d_{p}}} \delta\left(p \in \mathcal{K}_{i}\right),
$$

where the indicator function $\delta(\boldsymbol{A})$ equals 1 when the condition $\boldsymbol{A}$ is true, and zero otherwise, $M$ is the number of training images, and $\mathcal{K}_{i}$ is the kidney region in the training image $i$. We change the distance $d_{p}$ until we cover the whole distance domain available in the variability region. Then we multiply the histogram with kidney prior value which is defined as follows:

$$
\pi_{\mathcal{K}}=\frac{1}{M|\mathcal{V}|} \sum_{i=1}^{M} \sum_{p \in \mathcal{V}} \delta\left(p \in \mathcal{K}_{i}\right)
$$

We repeat the same scenario to get the marginal density of the background. The kidney and background distance empirical densities and the estimated Poisson distributions are shown in Fig 3 (a) and (b), respectively.

Distance Probabilistic Model: We define the shape penalty term $S\left(f_{p}\right)$ in Eq1 as $S\left(f_{p}\right)=-\ln P\left(d_{p} \mid f_{p}\right)$ where the distance marginal density of each class $P\left(d_{p} \mid f_{p}\right)$ is estimated as follow. Since each class $f_{p}$ does not follow perfect Poisson distribution, there will be a deviation between the estimated and the empirical densities. We model this deviation by a linear combination of discrete 
Gaussians with positive and negative components. So the distance marginal density of each class consists of a Poisson distribution and $K_{f_{p}}^{+}$positive and $K_{f_{p}}^{-}$ negative discrete Gaussians components as follows:

$$
P\left(d_{p} \mid f_{p}\right)=\vartheta\left(d_{p} \mid \lambda_{f_{p}}\right)+\sum_{r=1}^{K_{f_{p}}^{+}} w_{f_{p}, r}^{+} \varphi\left(d_{p} \mid \theta_{f_{p}, r}^{+}\right)-\sum_{l=1}^{K_{f_{p}}^{-}} w_{f_{p}, l}^{-} \varphi\left(d_{p} \mid \theta_{f_{p}, l}^{-}\right),
$$

where $\vartheta\left(d_{p} \mid \lambda_{f_{p}}\right)$ is a Poisson density with rate $\lambda, \varphi(. \mid \theta)$ is a Gaussian density with parameter $\theta \equiv\left(\mu, \sigma^{2}\right)$ with mean $\mu$ and variance $\sigma^{2} \cdot w_{f_{p}, r}^{+}$means the $r^{t h}$ positive weight in class $f_{p}$ and $w_{f_{p}, l}^{-}$means the $l^{\text {th }}$ negative weight in class $f_{p}$. This weights have a restriction $\sum_{r=1}^{K_{f_{p}}^{+}} w_{f_{p}, r}^{+}-\sum_{l=1}^{K_{f_{p}}^{-}} w_{f_{p}, l}^{-}=1$. We estimate the Poisson distribution parameter using the maximum likelihood estimator (MLE). To estimate the parameters of Gaussians components, we used the modified EM algorithm [11] to deal with the positive and negative components. Fig[3. (c) and (d) illustrate the probabilistic models components for kidney and background, respectively. The empirical and the final estimated densities are shown in Fig 3 (e) for the kidney and (f) for the background.

\subsection{Image Appearance Models}

Image appearance models are the gray level probabilistic model and the spatial interaction model.

A- Gray Level Probabilistic Model: To compute the data penalty term $D\left(f_{p}\right)$, we use the modified EM to approximate the gray level marginal density of each class $f_{p}$ using a LCG with $C_{f_{p}}^{+}$positive and $C_{f_{p}}^{-}$negative components. Similar to distance probabilistic model the gray level probabilistic model is defined as follows:

$$
P\left(I_{p} \mid f_{p}\right)=\sum_{r=1}^{C_{f_{p}}^{+}} w_{f_{p}, r}^{+} \varphi\left(I_{p} \mid \theta_{f_{p}, r}^{+}\right)-\sum_{l=1}^{C_{f_{p}}^{-}} w_{f_{p}, l}^{-} \varphi\left(I_{p} \mid \theta_{f_{p}, l}^{-}\right) .
$$

B- Spatial Interaction Model:The pairwise interaction model which represents the penalty for the discontinuity between pixels $p$ and $q$ is defined as follows:

$$
V\left(f_{p}, f_{q}\right)=\gamma \delta\left(f_{p} \neq f_{q}\right) .
$$

In this work we use a new analytical approach [11] to estimate the spatial interaction parameter $\gamma$. The simplest model of spatial interaction is the Markov Gibbs random field (MGRF) with the nearest 4-neighborhood. In the proposed approach, Gibbs potential is obtained analytically using MLE for a MGRF. The potential interaction is given by the following equation:

$$
\gamma=\frac{K^{2}}{K-1}\left(f_{\text {neq }}(\mathbf{f})-\frac{1}{K}\right)
$$

where $K=2$ is the number of classes in the image and $f_{\text {neq }}(\mathbf{f})$ denotes the relative frequency of the not equal labels in the pixel pairs. 


\subsection{Graph Cuts Optimal Segmentation}

To segment a kidney, we construct a graph (e.g. Fig4) and define the weight of each edge as shown in table 1 Then we get the optimal segmentation boundary between the kidney and its background by finding the minimum cost cut on this graph. The minimum cost cut is computed exactly in polynomial time for two terminal graph cuts with positive edges weights via $s / t$ Min-Cut/Max-Flow algorithm [12.

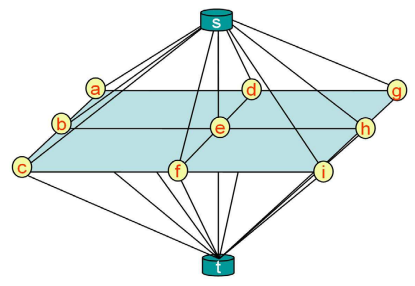

Fig. 4. Example of graph that used in image segmentation
Table 1. Graph Edges Weights

\begin{tabular}{|c|c|c|}
\hline Edge & Weight & for \\
\hline$\{p, q\}$ & $V\left(f_{p}, f_{q}\right)$ & $\{p, q\} \in \mathcal{N}$ \\
\hline \multirow{3}{*}{$s, p$} & $-\ln \left[P\left(I_{p} \mid 1\right) P\left(d_{p} \mid 1\right)\right]$ & $p \in \mathcal{V}$ \\
& $\infty$ & $p \in \mathcal{K}$ \\
& 0 & $p \in \mathcal{B}$ \\
\hline \multirow{3}{*}{$p, t$} & $-\ln \left[P\left(I_{p} \mid 0\right) P\left(d_{p} \mid 0\right)\right]$ & $p \in \mathcal{V}$ \\
& 0 & $p \in \mathcal{K}$ \\
& $\infty$ & $p \in \mathcal{B}$ \\
\hline
\end{tabular}

\section{Experiments}

Our proposed kidney segmentation framework is tested on a data set of DCEMRI of human kidney. To segment a kidney slice, we will follow the following scenario. The given image is aligned with the aligned training images. The gray level marginal densities of the kidney and its background are approximated using the proposed LCG model with positive and negative components. Fig 5 (a) shows the original image, (b) shows the aligned image, (c) illustrates the empirical densities as well as the initial estimated density using dominant modes in the LCG model, (d) illustrates the LCG components, (e) shows the closeness of the final gray level estimated density and the empirical one. Finally, (f) shows the marginal gray level densities of the object and back ground with the best threshold. To illustrate the closeness of the gray level between the kidney and its background, ( $\mathrm{g}$ ) shows the segmentation using gray level threshold $=72$. To emphasize the accuracy of the proposed approach, (h) shows the segmentation using the graph cuts technique without using the shape constraints (all the tlinks weights will be $-\ln P\left(I_{p} \mid f_{p}\right)$ ), and (i) shows the results of the proposed approach.

Samples of the segmentation results for different subjects are shown in Fig 7 , (a) illustrates the input images, (b) shows the results of graph cuts technique without shape constraints, and the results of the proposed approach are shown in (c).

Evaluation: to evaluate the results we calculate the percentage segmentation error from the ground truth (manual segmentation produced by an expert) as follows: 


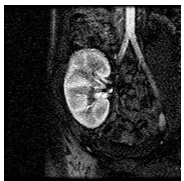

(a)

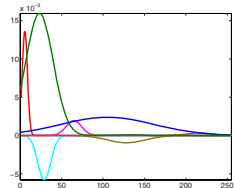

(d)

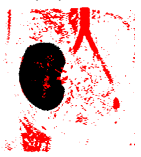

(g)

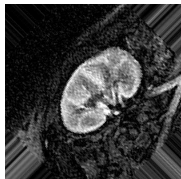

(b)

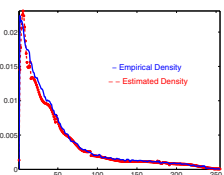

(e)

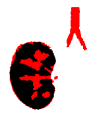

(h)

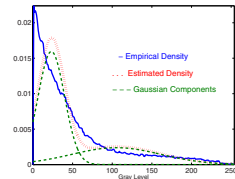

(c)

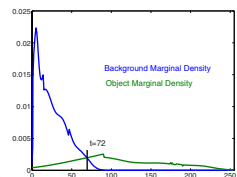

(f)

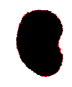

(i) (a)

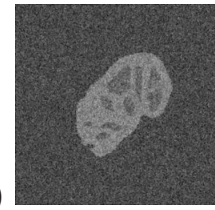

(b)
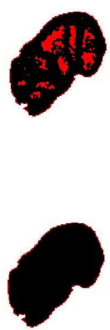

(c)

Fig. 6. Phantom Results (a) The phantom (b) Results of graph cuts without shape constraints $19.54 \%$ (c) Proposed approach results $0.76 \%$.

$$
\text { error } \%=\frac{100 * \text { Number of misclassified pixels }}{\text { Number of Kidney pixels }}
$$

For each given image, the binary segmentation is shown as well as the percentage segmentation error. The misclassified pixels are shown in red color. The statistical analysis of 33 slices, which are different than the training data set and for which we have their ground truths, is shown in table 2. The unpaired $t$-test is used to show that the differences in the mean errors between the proposed segmentation, and graph cut without shape prior and the best threshold segmentation are statistically significant (the two-tailed value $\mathbf{P}$ is less than 0.0001 ).

\section{Validation}

Due to the hand shaking errors, it is difficult to get accurate ground truth from manual segmentation. Thus to evaluate our algorithm performance, we have created a phantom shown in Fig [6(a) with topology similar to the human kidney. Furthermore, the phantom mimics pyramids that exist in any kidney. The kidney, the pyramids and the background signals for the phantom are generated according to the distributions shown in Fig $5(f)$ using the inverse mapping 
(a)
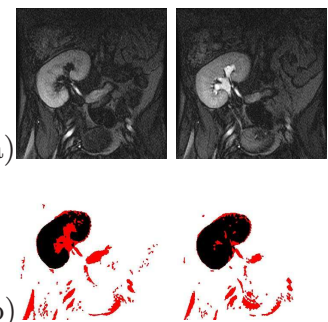

$74 \%$

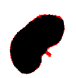

$52 \%$

(c)

$5.1 \%$
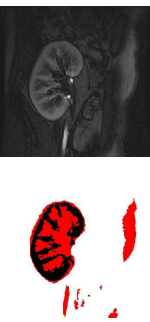

$77.4 \%$

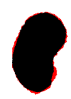

$6.7 \%$
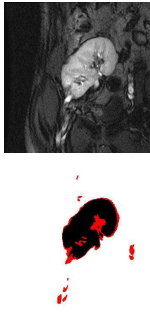

$26.4 \%$

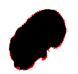

$5.5 \%$

Table 2. Accuracy of our segmentation on 33 slices in comparison to Graph Cut without shape and THreshold technique

\begin{tabular}{|l|c|c|c|}
\cline { 2 - 4 } \multicolumn{1}{c|}{} & \multicolumn{3}{c|}{ Algorithm } \\
\hline Error\% & Our & GC & TH \\
\hline Min. & $\mathbf{4 . 0}$ & 20.9 & 38.4 \\
Max. & $\mathbf{7 . 4}$ & 108.5 & 231.2 \\
Mean & $\mathbf{5 . 7}$ & 49.8 & 128.1 \\
Std. & $\mathbf{0 . 9}$ & 24.3 & 55.3 \\
\hline Significance, $\mathbf{P}$ & $<0.0001$ & $<0.0001$ \\
\hline
\end{tabular}

Fig. 7. More segmentation results (a)Original images (b) Results of Graph cuts without shape constraints (c) Proposed approach results.

methods. Fig $6(b, c)$ show our approach is almost 26 times more accurate than the graph cuts technique without shape constraints.

\section{Conclusions}

This paper proposed a new kidney segmentation approach that used graph cuts to combine region and boundary properties of segments as well as shape constraints. Shape variations were estimated using a new distance probabilistic model. The given image appearance models: image signal was approximated with a LCG with positive and negative components and the spatial interaction model was estimated using a new analytical approach. To get the optimal segmentation, we formulated a new energy function using the image appearance models and shape constraints and globally minimized this function using $s / t$ graph cuts. Experimental results showed that the shape constraints overcame the gray level inhomogeneities problem and precisely guided the graph cuts to accurate segmentations (with mean error $5.7 \%$ and standard deviation $0.9 \%$ ) compared to graph cuts without shape constraints (mean error $49.8 \%$ and standard deviation $24.3 \%$ ).

Acknowledgement. This work is supported by the Kentucky Lung Cancer Program and the NSF Egypt program.

\section{References}

1. de Priester, J., Kessels, A., Giele, E., den Boer, J., Christiaans, M., Hasman, A., van Engelshoven, J.: MR renography by semiautomated image analysis: performance in renal transplant recipients. J. Magn. Reson Imaging 14(2), 134-140 (2001) 
2. Giele, E.: Computer methods for semi-automatic MR renogram determination. PhD thesis, Eindhoven University of Technology, Eindhoven (2002)

3. Boykov, Y., Lee, V.S., Rusinek, H., Bansal, R.: Segmentation of dynamic N-D data sets via graph cuts using markov models. In: Niessen, W.J., Viergever, M.A. (eds.) MICCAI 2001. LNCS, vol. 2208, pp. 1058-1066. Springer, Heidelberg (2001)

4. Sun, Y., Jolly, M.P., Mourua, J.M.: Integrated registration of dynamic renal perfusion MR images. In: Proce. IEEE ICIP, pp. 1923-1926. IEEE Computer Society Press, Los Alamitos (2004)

5. Rousson, M., Paragios, N.: Shape priors for level set representations. In: Heyden, A., Sparr, G., Nielsen, M., Johansen, P. (eds.) ECCV 2002. LNCS, vol. 2351, pp. 78-92. Springer, Heidelberg (2002)

6. Leventon, M.E., Grimson, W.E.L., Faugeras, O.: Statistical shape influence in geodesic active contours. In: Proc. IEEE CVPR, p. 1316. IEEE Computer Society Press, Los Alamitos (2000)

7. Chen, Y., Thiruvenkadam, S., Huang, F., Wilson, D., Geiser, E.A., Tagare, H.D.: On the incorporation of shape priors into geometric active contours. In: IEEE VLSM, pp. 145-152. IEEE Computer Society Press, Los Alamitos (2001)

8. Huang, X., Metaxas, D., Chen, T.: Metamorphs:deformable shape and texture models. In: Proce. IEEE CVPR, pp. 496-503. IEEE Computer Society Press, Los Alamitos (2004)

9. Paragios, N.: A level set approach for shape-driven segmentation and tracking of the left ventricle. IEEE Trans. Medical Imaging 22, 773-776 (2003)

10. Viola, P.: Alignment by Maximization of Mutual Information. PhD thesis, Massachusetts Inst. of Technology, Cambridge, MA (1995)

11. Farag, A., El-Baz, A., Gimel'farb, G.: Pecise segmentation of multimodal images. IEEE Trans. Image Processing 15(4), 952 (2006)

12. Boykov, Y., Kolmogorov, V.: An experimental comparison of min-cut/max-flow algorithms for energy minimization in vision. IEEE Trans. PAMI 26(9), 1124 (2004) 